\title{
Métodos Axiomáticos: a Interpretação Matemática de Lawvere da Lógica de Hegel
}

\author{
Axiomatic Methods: \\ Lawvere's Mathematical Interpretation of Hegel's Logic \\ Nicholas Kluge Corrêa \\ (Pontifícia Universidade Católica do Rio Grande do Sul, Brasil)
}

\section{Resumo}

O pensamento axiomático de Hilbert foi um influente modelo que motivou vários movimentos no início do século $X X$. O formalismo axiomático fornece, através do uso da lógica de primeira ordem, uma importante fundação para modelos lógicos formais, o que, para Hilbert, representaria um modelo universal de investigação empírica, não só para a matemática, mas para todas as ciências naturais. Contudo, no caso mais específico da matemática, existe uma certa descomunicação entre os fundamentos da matemática e sua prática, onde métodos informais, ainda promovem elegantes ferramentas para matemáticos de diversas áreas. É exatamente esta assincronia entre os fundamentos da matemática, e a sua prática que iremos investigar neste estudo. Lawvere, insatisfeito com a "fundação não fundamentada" do método axiomático proposto por Hilbert, e inspirado pela dialética hegeliana, procurou revisar os fundamentos da matemática pela lógica categórica e a Teoria das Categorias. Vemos neste estudo, como as interpretações de Lawvere de conceitos da lógica de Hegel, como, equivalência, unidade dos opostos e "aufheben", permitem uma nova abordagem matemática, com um posicionamento filosófico que procura, de certa forma, transcender a dicotomia entre escolas analíticas e continentais. Lawvere trata a lógica objetiva de Hegel como uma possível estratégia para resolver o problema de aterramento lógico em metafísica. Por fim, vemos como as contribuições de Lawvere para a axiomatização da lógica categórica tiveram impactos inovadores na metamatemática, especialmente no desenvolvimento das fundações univalentes de Vladimir Voevodsky.

Palavras-chave: Métodos Axiomático. Lawvere. Hegelianismo. Categorias. Homotopia.

\begin{abstract}
Hilbert's axiomatic thinking was an influential model that motivated several movements in the early 20th century. Axiomatic formalism provides, through the use of first-order logic, an important foundation for formal logical models, which, for Hilbert, would represent a universal model of empirical research, not only for mathematics, but for all natural sciences. However, in the most specific case of mathematics, there is a certain lack of communication between the foundations of mathematics and its practice, where informal methods still promote elegant tools for mathematicians from different areas. It is exactly this asynchrony between the foundations of mathematics and its practice that we will investigate in this study. Lawvere, dissatisfied with the "unfounded foundation" of the axiomatic method proposed by Hilbert, and inspired by the Hegelian dialectic, sought to review the foundations of mathematics by categorical logic and Category Theory. We see in this study, how Lawvere's interpretations of concepts from Hegel's logic, such as equivalence, unity of opposites and "aufheben", allow a new mathematical approach, with a philosophical position that seeks, in a way, to transcend the dichotomy between schools analytical and continental. Lawvere treats Hegel's objective logic as a possible strategy to solve the problem of logical grounding in metaphysics. Finally, we see how Lawvere's contributions to the axiomatization of categorical logic had innovative impacts on metamathematics, especially on the development of Vladimir Voevodsky's univalent foundations.
\end{abstract}

Keywords: Axiomatic Methods. Hegelianism. Lawvere. Categories. Homotopy. 


\section{Método axiomático na visão de Hilbert}

O método axiomático, também conhecido como o "Pensamento Axial" foi um modelo filosófico e matemático defendido proeminentemente por David Hilbert (1996), onde, para Hilbert: o conceito de axiomatização, em termos epistemológicos, seria logicamente, e incontestavelmente, a única forma de "pensar com consciêncid", ou seja, racionalizar. Nas palavras de Hilbert:

Se considerarmos mais de perto uma teoria em particular, vemos sempre que algumas propostas básicas [...] estão subjacentes à construção do quadro de conceitos, e estas propostas são então processadas por elas próprias para a construção, de acordo com princípios lógicos, de todo o quadro/teoria. Estas proposições fundamentais podem ser consideradas [...] como os axiomas de uma teoria do conhecimento: o desenvolvimento progressivo de cada proposição do conhecimento reside então apenas na construção lógica do quadro de axiomas já pressupostos. Este ponto de vista é especialmente predominante na matemática pura. [...] Qualquer coisa que possa ser objeto do pensamento científico torna-se dependente do método axiomático, e assim indiretamente da matemática (HILBERT, 1996, p. 1108-1115).

A ideia de lógica de Hilbert pode ser interpretada como a concatenação de proposições, cada uma derivada das anteriores até a base axiomática do sistema, seus prioris, em conformidade com as regras de um sistema lógico. Dessa forma, o pensamento axiomático é uma forma de veículo que permite o pensador externalizar o pensamento em uma linguagem adequada, e 
supostamente "livre" de ambiguidade, chegando assim as implicações que tais premissas levam o pensamento racional a chegar (BOUBRBAKI, 1950). Dessa forma o formalismo axiomático fornece aquilo que apenas pela utilização da lógica não poderíamos alcançar, dando uma importância a fundamentação da lógica a axiomas.

A ideia informal do pensamento axiomático é de que: axiomas são importantes, sem eles, não sabemos sobre "o que" estamos falando. Axiomas nos permitem restringir o espaço de possibilidade, por exemplo: através dos axiomas da Peano Aritmética, conseguimos definir o que são os números naturais: definindo o como um número natural, sendo o único que não possui um sucessor (S), e a sucessão de "entidades" após 0, informalmente da seguinte forma: o número 1 pode ser definido como $S(0), 2$ como $S(S(0)$ ) (que também é $S(1)$ ) e, no geral, qualquer número natural n como $S^{n}(0)$. em outras palavras, $\{0, S(0), S(S(0)), \ldots\} \subseteq \mathbb{N}$, onde todos os números naturais estão contidos em $\mathbb{N}$ (PEIRCE, 1881).

O entusiasmo de Hilbert pelo Método Axiomático, por mais que não seja universalmente aceito, ainda é o padrão que molda a noção moderna da teoria axiomática, e sua Grundlagen der Geometrie (Fundamentos da Geometria) ainda é considerado como um dos paradigmas para os fundamentos da matemática e a filosofia da matemática (HILBERT, 1899). Em comparação com outros modelos propostos, a filosofia matemática de Hilbert, denominada Formalismo, distingue-se do, por exemplo, intuicionismo de Brouwer, e do racionalismo de Russell. Podemos nos perguntar: o quão bem o método axiomático tem impactado e contribuído a matemática prática? Por um lado, formalismo de Hilbert continua a ser o método padrão de construção de teorias físicas, 
filosóficas e matemáticas, contudo, tanto na matemática quanto nas ciências naturais, diversos avanços nestas áreas permanecem sendo feitos por métodos "informais", ou seja, de certa formas que escapam (inteligentemente) dos métodos lógicos formais axiomáticos (RODIN, 2012).

Atualmente, existe uma situação um tanto quanto paradoxal: Hilbert em suas Fundações da Matemática (1967) sugeriu que seu Método Axiomático Formal fosse usado como um instrumento básico de toda pesquisa científica, e inclusive filosófica. Quando falamos de métodos axiomáticos contemporâneos nos referimos a Teoria de Conjuntos Axiomática, e uma das críticas levantadas contra o este método é: o Método Axiomático importa apenas nos fundamentos, ou seja, na meta-teoria, enquanto a ciência convencional se preocupa muito pouco com seus próprios fundamentos. Dessa forma a questão dos fundamentos da matemática, por exemplo, fica ao encargo de lógicos e filósofos, enquanto a realização da matemática em si a matemáticos.

Esta aparente assincronia entre os fundamentos da matemática e a prática da matemática, pode soar como insatisfatória ao leitor, e de fato, a muitos outros teóricos, matemáticos e filósofos. Uma noção de fundamento mais satisfatória é descrita por Lawvere e Rosebrugh (2003) da seguinte forma:

Uma fundação explicita as características gerais essenciais, uture ents e operações de uma ciência, assim como as suas origens e leis gerais de desenvolvimento. O propósito de torná-las explícitas é fornecer um guia para o aprendizado, uso e desenvolvimento uture da ciência. Uma fundação "purd" que esquece este propósito e persegue uma fundação "especulativa" por si mesma, é 
Para tornar mais claro as limitações do método axiomático é interessante analisarmos situações onde este falha em prover uma resposta satisfatória. Desde o trabalho revolucionário de Zermelo (1903) que futuramente deu origem a Teoria dos Conjuntos Axiomática (Teoria dos conjuntos Zermelo-Fraenkel), a matemática moderna vem usando este formalismo e linguagem como a base para sua identidade, o que torna a teoria dos conjuntos um exemplo de uma teoria desenvolvida totalmente dentro de um cenário axiomático formal. Um famoso exemplo de um problema que ilude o formalismo de Zermelo-Fraenkel é um dos 23 problemas listados por Hilbert (1902), e considerado por ele como o mais importante, a Hipótese Contínua de Cantor (HC) (1892).

\section{Hipótese Contínua}

A HC é um problema matemático onde até hoje existe uma certa ambiguidade sobre se o problema foi "realmente" resolvido ou não. A Hipótese Continua, diz respeito a cardinalidade, o número de elementos dentro de um conjunto, e se existe uma bijeção, uma correspondência um-para-um entre os elementos de dois conjuntos diferentes (COHEN, 2008). Intuitivamente, dois conjuntos $X$ e $Y$ tem a mesma cardinalidade se é possível "emparelhar" os elementos de $X$ com elementos de $Y$ de forma que cada elemento de $X$ corresponde com exatamente um elemento de $Y$, e vice-versa. O conjunto $\{$ maçã, melancia, laranja\} tem a mesma cardinalidade que \{quadrado, triângulo, círculo\}. A HC procura responder a 
pergunta de se é sempre possível encontrar uma bijeção entre conjuntos com infinitos elementos. Por exemplo, o conjunto dos números Naturais, tem a mesma cardinalidade que os número Inteiros, e o mesmo corresponde para os números Racionais, existindo uma correspondência um-para-um entre os conjuntos citados, todos tendo a mesma cardinalidade, todos sendo contáveis, por exemplo:

$$
\begin{gathered}
\mathbb{N}=1,2,3,4,5,6,7,8,9, \ldots \rightarrow \infty \\
\mathbb{Z}=1,-1,2,-2,3,-3,4,-4,5, \ldots \rightarrow \infty
\end{gathered}
$$

Ambos os conjuntos, $\mathbb{N}$ (Naturais) e $\mathbb{Z}$ (Inteiros) podem ser listados e pareados por uma bijeção, portanto, ambos os conjuntos infinitos possuem a mesma cardinalidade, ambos possuem o mesmo "tipo" de infinidade.

Contudo, existe uma bijeção entre os números Naturais e os números Reais? Esta é a pergunta que inspirou Cantor a conjecturar sua Hipótese Contínua. Através de seu argumento diagonal Cantor provou que a cardinalidade do conjunto de números Naturais é estritamente menor que a do conjunto de números Reais. Porém, a prova de Cantor não indica a medida em que a cardinalidade dos números Naturais é menor do que a dos números Reais. Dessa forma Cantor propôs a Hipótese Contínua, como uma possível solução para esta questão (CANTOR, 1892), afirmando: Não há nenhum conjunto cuja cardinalidade esteja estritamente entre a dos números Naturais e a dos números Reais.

\section{Argumento Diagonal de Cantor}

O método diagonal de Cantor é uma ferramenta elegante e poderosa, que influenciou diversas outras 
importantes provas em áreas como lógica e metamatemática, como, os Teoremas de Incompletude de Gödel, e a incomputabilidade do Problema de Parada de Turing. Mostraremos abaixo diretamente o método, que através de sua compreensão, permite a extensão para os demais problemas e paradoxos citados.

Primeiramente, assumimos que os números Reais podem ser pareados em uma correspondência uma-paraum com os números Naturais, o que nos levará a uma contradição. Suponha que possamos listar todos os números entre 0 e 1 que podem ser representados por decimais (o método funciona para qualquer base numérica), como, por exemplo, 0,25, ou decimais que repetem infinitamente como, por exemplo, 0,123123123... Por conveniência usaremos representações infinitas em todos os casos, de modo que anexamos um número infinito de zeros no final de cada número Real cessante. Agora suponha que listássemos todos os números Reais, todas as infinitas representações decimais:
0.0000000000000 ..
0.1000000000000 ..
0.5000000000000 ..
0.3333333333333 ..
0.6666666666666 ..
0.2500000000000 ..
0.7500000000000 .
0.2000000000000 ..
0.4000000000000 ..
0.6000000000000 ..
0.8000000000000 ..
0.1666666666666 ..
$0.8333333333333 .$.

Imagine que está lista é infinitamente longa, e contém todas as representações decimais de números 
Reais possíveis. Agora manipulamos a diagonal ressaltada em vermelho dessa lista. A diagonalização de Cantor nos mostra como construir um número fora desta lista, dígito por dígito, uma representação que não está na lista, mesmo ela sendo supostamente a lista de "todos os números Reais possíveis". Existem vários procedimentos para alcançar o mesmo resultado, contudo, usaremos o seguinte algoritmo: localize o i-ésimo número Real e sua iésima representação decimal, em seguida adicione "1" a este número, se ele for um "9", transforme o número em um "0". Este procedimento produziria o número Real:

\subsection{4...}

O número produzido não pode ser encontrado em nenhuma posição na lista. Por definição o número criado difere do primeiro número Real na primeira casa após o ponto decimal, difere do segundo número Real na segunda casa após o ponto decimal, e etc. Porém, a lista deveria conter todos os números Reais, nós poderíamos simplesmente adicionar o número criado na lista. Contudo, a diagonalização de Cantor pode simplesmente ser repetida de novo, criando números que, a princípio, não estão listados na lista de todos os números Reais. $O$ que nos leva a uma contradição, nosso erro foi em presumir que os números Reais poderiam ser listados, e eles não podem, pois, são incontáveis, inumeráveis.

Cantor (1892) usou este método para provar o resultado de que os números Reais não são contáveis, e que nenhuma lista infinita de decimais pode conter todos os seus elementos. Assim, qualquer lista desse tipo deve estar ncomplete, não existindo uma bijeção entre os números Reais e os números Naturais (os números Inteiros e os Racionais também), tornando estes conjuntos 
cardinalmente diferentes. Formalmente, a cardinalidade dos números Reais é muito, muito maior do que a dos números Naturais:

\section{$\mathbb{R} \gg \mathbb{N}$}

Este resultado causou uma divisão no sistema axiomático de Zermelo-Fraenkel, pois, em 1938 Gödel (1938) provou que o sistema axiomático de ZermeloFraenkel é consistente com a HC. Porém, em contraponto, em 1963 Cohen (1963) provou que a teoria dos conjuntos Zermelo-Fraenkel também é consistente com a negação da HC. Portanto, o que é aceito modernamente é que a hipótese contínua de Cantor, ou a sua negação, podem ser derivados dos axiomas de Zermelo-Fraenkel, sendo uma questão de total ambiguidade, dependendo de qual modelo de Zermelo-Fraenkel você quer adotar (KUNEN, 1980). Este resultado controverso mostra como a HC é independente dos próprios fundamentos da matemática, o que torna tais fundações questionáveis. Talvez novos axiomas, ou algum sistema de axiomas totalmente novo, possa eventualmente ajudar a estabelecer a verdade ou negação da HC. Contudo, tal feito ainda permanece em aberto.

No modelo axiomático proposto por Hilbert, como Zermelo-Fraenkel, a única coisa que a matemática pode fazer de acordo com este ponto de vista é: fornecer propostas verdadeiras do tipo, se - então, se as propostas dedutíveis são verdadeiras dado os axiomas do sistema em questão. A ausência de dialética torna este modelo incompatível não só com a prática matemática comum, mas, com prática da metamatemática, como no exemplo da HC, pois, sua proposição não é expressa na forma "seentão", mas sim como uma verdade "absolutd", que não se 
refere a nenhuma estrutura formal em particular. Dessa forma, o método axiomático de Hilbert não é capaz de diferenciar entre uma teoria axiomática que contém um conhecimento valioso de teorias tautológicas cíclicas sem nenhum valor epistêmico. Com certeza, o progresso feito por Hilbert permitiu grande progresso em teorias matemáticas construídas com sistemas formais lógicos, contudo, ele não serve, como Hilbert sugeriu, como uma base que fundamente a construção de todas as teorias científicas, e particularmente como fundamentos da matemática e filosofia.

Neste estudo revisaremos o trabalho do matemático William Lawvere, que inspirado pela lógica e dialética de Hegel, procurou resolver o problema dos fundamentos da matemática com a utilização da lógica categórica e a Teoria das Categorias.

\section{Novas Formas de Lógica}

Com a ascensão da filosofia analítica no século XX, e a adoção, e desenvolvimento da nova lógica forjada por Frege e Peano, veio uma rejeição radical do paradigma filosófico existente, o idealismo de Hegel. O entendimento de Hegel sobre o termo "Logik" é muito mais amplo do que normalmente é o caso, e sua "lógica objetiva" da Ciência da Lógica foi considerada uma espécie de metafísica pelo movimento positivista. Um exemplo desta crítica positivista a metafísica foi o movimento conhecido como o Círculo de Viena, onde, buscava-se reconceituar o empirismo a partir das novas descobertas científicas e demonstrar as falsidades da Metafísica. O positivismo lógico, mais tarde chamado de empirismo lógico, e também conhecido como neopositivismo, foi um movimento filosófico cuja tese central foi o princípio da 
verificação, o verificacionismo, onde se afirmava que apenas as declarações verificáveis através da observação direta ou da prova lógica são significativas, descartando a metafísica como sem sentido (CARNAP, 1932).

Bertrand Russell foi categórico ao afirmar que a nova lógica quebraria o paradigma filosófico estabelecido pelo idealismo lógico de, por exemplo, Kant e Hegel, no final de seu livro "Logic As The Essence Of Philosophy":

A velha lógica coloca o pensamento em grilhões, enquanto a nova lógica lhe dá asas. Ela introduziu, em minha opinião, o mesmo tipo de avanço na filosofia que Galileu introduziu na física, tornando finalmente possível ver que tipos de problemas podem ser capazes de solução, e que tipos estão além dos poderes humanos. E onde uma solução parece possível, a nova lógica fornece um método que nos permite obter resultados que não apenas incorporam idiossincrasias pessoais, mas devem comandar o assentimento de todos os que são competentes para formar uma opinião (SULLIVAN, 2003, p. 277).

A doutrina positivista é caracterizada como uma realizar uma das críticas mais fortes ao trabalho e filosofia de Hegel. Em 1951, Hans Reichenbach escreveu:

Hegel foi chamado o istema e de Kant; isso é um grave mal-entendido de Kant e uma elevação injustificada de Hegel. $O$ istema de Kant, embora provado insustentável por desenvolvimentos istema $e$, foi a istema e de uma grande mente de estabelecer 0 racionalismo istem base científica. O istema de Hegel é a pobre construção de um fanático que viu uma verdade empírica e tenta torná-la uma lei 
lógica, dentro da mais não-científica de todas as lógicas. Enquanto o istema de Kant marca o auge da linha histórica do racionalismo, o istema de Hegel pertence ao período de decadência da filosofia especulativa que caracteriza o século XIX. (REICHENBACH, 1951, p. 72)

Embora a filosofia analítica tenha rejeitado a metafísica e lógica de Hegel em favor da análise da lógica matemática, em particular a lógica proposicional, recentes desenvolvimentos nos fundamentos da matemática através da Teoria das Categorias, utilizam da lógica hegeliana para sugerir uma nova forma de pensamento matemático, não pela na lógica proposicional, mas pela lógica modal.

Lógica modal refere-se a um enriquecimento da lógica proposicional onde as operações padrão $(\&, \neg, \rightarrow, \equiv$, e etc) são acompanhadas por certas operações extras, chamados operadores modais, denotados por “ $\square$ " ou símbolos similares. Assim, uma proposição " $\square P^{n}$ é uma proposição nova cuja interpretação depende do tipo de operador modal, como, por exemplo: " $P$ é possivelmente verdadeiro", ou "P se tornará eventualmente verdadeiro", ou "P poderia ser verdadeiro" (GARSON, 2018). Não há nenhum axioma estabelecido o que um operador modal deve satisfazer em proposições, permitindo uma grande flexibilidade e aplicabilidade da lógica modal.

No século $X X$, a ascensão da lógica modal permitiu um ressurgimento da metafísica analítica, como, por exemplo: na teoria de contrafactualidade de Lewis, também conhecida como modelo de mundos possíveis de Lewis-Stalnaker (LEWIS, 1973,), uma proposição lógica consiste em sua realização em todos os mundos possíveis 
vizinhos (similares/mais próximos), e através de um operador modal, " $>$ ", onde $X>Y$ significa, "Se fosse $X$, então seria $Y^{\prime \prime}$, traz a possibilidade de trazer significado a esses mundos possíveis. Atualmente, tópicos da metafísica analítica incluem a causalidade, necessidade, espaço e tempo, identidade, só são expressados de forma formal, na filosofia, através da lógica modal, e não da lógica proposicional.

Dada as limitações da filosofia analítica, muito devido a sua fundamentação na lógica proposicional de primeira ordem, e, apesar da longa rejeição inicial das ideias Hegelianas, recentemente os escritos de Hegel vêm sido colocados sobre uma nova perspectiva, como, por exemplo, pelo matemático Francis William Lawvere (Muncie, Indiana, 9 de fevereiro de 1937). Lawvere é um matemático estadunidense conhecido por seu trabalho em teoria das categorias, topos e filosofia da matemática, onde inspirado pelo conceito de "Unidade dos Opostos" presente na lógica de Hegel, buscou formalizar a lógica categórica pela dialética da lógica de Hegel. Uma ligação que levou a lógica modal e a teoria das categorias a dar surgimento a Teoria dos Tipos Homotópica, uma teoria que promete modificar as fundações da matemática (LAWVERE, 1991).

\section{Da Tríade Dialética em Kant para a Aufhebung em Hegel}

A “Wissenschaft der Logik”, Ciência da Lógica, (1 ${ }^{a}$ ed. 1812-1816, HEGEL, 2010) pode ser considerada como um dos principais textos da filosofia de Hegel, como a "Phänomenologie des Geistes", Fenomenologia do Espírito. A Ciência da Lógica tem de ser vista no contexto da filosofia do início do século XIX, sendo uma forma de 
resposta a "refundação" da metafísica por Kant. O sistema idealista proposto por Kant, por mais que tenha mudado as visões metafísicas e epistemológicas da época, ele deixou a lógica tradicional intocada, e precisamente neste ponto que Hegel se propõe a estender o exame crítico dos fundamentos do conhecimento iniciado por Kant à própria lógica.

A "redescoberta da tríade dialética", como Hegel atribui a Kant (1763), fornece um conceito preliminar a distinção entre contradições e oposições reais, algo que antecipa a divisão analítico-sintética de Hegel, a tríade dialética: dividida em si (sujeito), para si (objeto), e em sipara si (objeto, tal como experienciado pelo sujeito, e sujeito, refletido a si mesmo ao experienciar o objeto) (HEGEL, 2010). A tríade dialética aqui origina o termo "Aufhebung", à síntese de oposições, conceito hegeliano expresso no uso dos termos "tese", "antítese" e "síntese".

Mesmo que Kant tenha organizado a síntese dos termos em positivos e negativos, algo que paraKant causaria a razão a ficar necessariamente enredada nas contradições da dialética transcendental por sua própria natureza, para Hegel está interpretação pouco conclusiva seria um indicador do papel positivo das contradições como aspecto essencial do pensamento. Aqui a dialéctica e a Aufhebung entram em cena, pois, a crítica de Hegel a Kant seria que, na verdade, as categorias não são dadas aprioristicamente, mas sim como algo resultado da contínua transformação, do se-tornar. Assim, a lógica deixa de ser um inventário de categorias e torna-se um sistema de transformações de categorias. Curiosamente, esta terminologia Hegeliana seria apropriada pela matemática no estudo formal de categorias no século XX (EILENBERG, MACLANE, 1945). Hegel explica o conceito de 
Aufheben (HEGEL, 2010, p.113) como um dos mais importantes em toda a filosofia, se repetindo constantemente em todos os lugares, como, por exemplo, no conceito de "omnis determinatio est negatio" de Spinoza, para Hegel, Aufhebung é o modo desta coexistência de negação-afirmação.

Aufhebung é um conceito extremamente abrangente, que constitui uma das edificações centrais da tese hegeliana, e para Lawvere, este conceito propõe introspecções interessantes para a matemática.

\section{A interpretação de Lawvere da lógica objetiva de Hegel}

Lawvere (2000) sugere um exemplo particularmente simples onde a ideia de equivalência é dada uma interpretação dialética, permitindo um novo significado de "equivalêncid", dentro da teoria categórica.

Que $\mathbb{N}$ sejam os números naturais $\{0,1, \ldots\}$ vistos como uma categoria através da sua habitual ordenação. Que $E, D: N \rightarrow \mathbb{N}$ sejam os dois functores, um functor é um mapeamento entre categorias, um functor "par" e outro "ímpar", definidos por $E(n):=2 n$ e $D(n):=2 n+1$.

Ambas categorias correspondem a duas subcategorias de inclusões, $N$-pares e $N$-impares. Nessa situação ambas subcategorias se "opõem" uma à outra, $N$ - pares $\neq N$-impares, no entanto, são "idênticas", pois, existe uma bijeção, uma mapeamento um-para-um de $N_{\text {par }} \rightarrow \underline{\sim} N_{\text {ímpar }}$. Além disso, ambas são englobadas como parte de um todo, os números Naturais, cuja estrutura geral pode ser representado por ambos os functores paralelos:

$$
N_{\text {par }} \rightarrow \simeq \underline{N} \simeq \leftarrow N_{\text {impar }}
$$


Normalmente, e talvez analiticamente, conceitos opostos não são considerados equivalentes, mas nesta interpretação de equivalência como um mapeamento entre conjuntos (bijeção), ou em termos hegelianos, uma transformação, será sempre um par constituído por duas subcategorias, uma reflexiva e outra co-reflexiva. Lawvere (2000) sugere que se adicionarmos um terceiro functor que chamamos $T$, podemos encapsular as relações $E$ e $D$ formando um adjunto triplo $E \dashv T \dashv D$, onde este triplo expressa a unidade das subcategorias (co)reflexivas:

- $E \circ T \dashv D \circ T$ representa a oposição entre $E$ e $D$, e, ao mesmo tempo;

- $T \circ E \simeq T \circ D$ representa a identidade $E$ entre $D$ pela equivalência implicada.

Informalmente, $T$ une, se opõe e identifica $E$ e $D$ ao mesmo tempo.

O conceito de adjunção acima expressa uma dualidade, especificamente, uma dualidade entre opostos. O conceito de correspondência e equivalência em termos de adjuntos triplos foi sugerido por Lawvere (1991, p. 7; 1994 , p. 11) com o intuito de formalizar o conceito de "Unidade e Identidade dos Opostos" como aparecem informalmente na Ciência da Lógica de Hegel (HEGEL, 2010), e também, para captar a noção de dialética hegeliana.

\section{A "unidade dos opostos": aufhebung, e a lógica categórica de Lawvere}

Para entendermos a formalização de Lawvere do conceito de unidades de opostos, é importante revermos a tese de Hegel na Ciência da Lógica, onde Hegel fornece um relato de sua lógica objetiva da seguinte forma:

Ágora Filosófica, Recife, v. 20, n. 3, p. 206-239, set./dez., 2020 • 221 
O que estamos tratando em lógica não é um pensamento sobre algo que existe independentemente como base para o nosso pensamento e à parte dele, nem formas que supostamente fornecem meros sinais ou marcas distintivas da verdade; pelo contrário, as formas e autodeterminações necessárias do pensamento são o conteúdo e a verdade última em si(HEGEL, 1990, p.50).

Uma possível interpretação, é que Hegel busca uma lógica que raciocine sobre as coisas com as quais raciocina. E este conceito está na essência da lógica categórica, uma forma de lógica modal formal em matemática que permite o desenvolvimento de vários conceitos e aplicações para a Teoria das Categorias e Teorias dos Tipos, pois, nessas disciplinas não existe uma distinção entre os objetos da teoria (elementos, grupos, categorias, tipos, morfismos, funções) e a lógica (proposições e provas) utilizada para raciocinar sobre os objetos da teoria, algo conhecido com "proposições como tipos".

Lawvere (1991) faz essa comparação da lógica objetiva de Hegel com a lógica categórica matemática, onde a lógica toma a forma de ferramentas, que também podem ser consideradas elementos/categorias objetivas, uma forma de objeto que possui a funcionalidade. Nesta interpretação a verdade e falsidade não são considerados estados opostos e desconexos, mas as extremidades de uma transformação, a verdade sendo representada por um objeto terminal, e a falsidade por um objeto inicial. Estes princípios podem ser formalizados em termos de transformações (adjunções), como, no exemplo explicado acima, onde pares opostos são adjuntos à esquerda $(E)$ e 
à direita $(D)$ de um functor comum $(T)$ : falsidade e verdade são lados opostos ( $E$ e $D$ ) de um functor terminal $(T)$, e assim regressamos a Hegel. O princípio orientador fundamental da lógica objetiva é a "unidade dos opostos", Aufhebung se resume em "sublimar" uma oposição.

Para Hegel a ideia primária de oposição vem da unidade entre vacuidade, aplicando-se a nada, e tautologia, aplicando-se a qualquer coisa, a unidade entre o Nada e o Ser. Assim, na visão hegeliana, para o sujeito entreter qualquer ideia, é necessário poder entreter o seu oposto, caso contrário, a sua ideia é vazia no sentido de que poderia aplicar-se a qualquer coisa. Hegel explica da seguinte forma: falar até mesmo do Nada é considerá-lo como uma coisa, é fazê-lo ser, mesmo sendo algo sem características, um Ser puro, a falta de conteúdo implica a ausência de características, portanto, o Ser puro é o Nada (HEGEL, 2010, p. 59-60).

A interpretação da Lawvere (1991) dessa oposição pode ser interpretada ao representar o Nada como o objeto nulo inicial " $\varnothing$ " e o Ser como o objeto terminal "1". Em um espaço (topos) de categorias, " $\emptyset$ " é uma categoria vazia e "1" é um único ponto/elemento de uma categoria. Estes são opostos no sentido em que são distintos, porém, unificados no sentido em que são os adjuntos esquerdo $E$ e direito $D$ do mesmo functor $T$. Na Teoria das Categorias, a adjunção é uma relação que dois functores podem ter, quando nesta relação eles são chamados de functores adjuntos, sendo o adjunto esquerdo e o outro o adjunto direito (KAN, 1958).

Por exemplo, um adjunto entre as categorias $A$ e $B$ é um par de functores $E$ e $D$ :

$$
E: B \rightarrow A \text { e } D: A \rightarrow B
$$

e, para todos os objetos $X$ em $A$ e $Y$ em $B$, uma bijeção

Ágora Filosófica, Recife, v. 20, n. 3, p. 206-239, set./dez., 2020 • 223 
entre os respectivos conjuntos de morfismo:

\section{$\operatorname{hom}_{A}(E Y, X) \cong \operatorname{hom}_{B}(Y, D X)$}

de tal forma que esta família de bijeções, possui uma relação uma-para-um, entre os elementos $X$ e $Y$. O functor $E$ é chamado de functor adjunto esquerdo, ou simplesmente adjunto esquerdo a $D$, enquanto $D$ é chamado de functor adjunto direito a $E$.

Este formalismo lógico categórico é essencial para a definição de conceitos como "equivalência" nesta área da matemática, e a ideia de unidade entre as oposições de Lawvere é profundamente inspirada pela oposição hegeliana do Nada e o Ser. Assim como Hegel diz que não podemos pensar em algo sem o seu oposto, aqui recuperarmos o oposto da coisa em $\mathrm{Si}$, o Nada, os pontos opostos de uma transformação dialética, através da singularidade das adjunções.

\section{Lógica categórica e dialéctica Hegeliana}

A crítica de Lawvere ao Método Axiomático proposto por Hilbert é sustentado pela filosofia de Hegel, que, além do conceito de Aufhebung citado anteriormente, usa a distinção hegeliana entre a lógica objetiva e a subjetiva. A perspectiva hegeliana de Lawvere sobre a matemática, especificamente a teoria e lógica categórica, é crucial para uma compreensão filosófica da sua obra, e como Lawvere afirma, a apreciação da importância da dialéctica de Hegel na lógica categórica, não implica a necessidade de ser um "hegeliand". Lawvere afirma explicitamente que ele próprio não é hegeliano, mas que a importância do conceito de dialética como proposto por Hegel seria algo de valor intrínseco o suficiente para transcender a "contínua batalha" entre as tradições filosóficas analíticas e continentais. Nas palavras 
de Lawvere:

É minha convicção que, na próxima década e no próximo século, a técnica forjada pela teoria categórica será de valor para a filosofia dialéctica, emprestando preciosos formalismos com modelos matemáticos, auxiliando controversas distinções filosóficas antigas, tais como: geral vs. particular, objetivo vs. subjetivo, ser vs. tornar-se, espaço vs. quantidade, igualdade vs. diferença, quantitativo vs. qualitativo, etc... A explícita atenção dos matemáticos a tais questões filosóficas é necessária para alcançar o objetivo de tornar a matemática (portanto, outras ciências) mais amplamente aprendida e utilizável. É claro que isto exigirá que os filósofos aprendam matemática e que os matemáticos aprendam filosofia (LAWEVERE, 1992, p. 16).

Lawvere por ser primariamente um matemático, e secundariamente um filósofo, compartilha com outros filósofos, incluindo analíticos como Russell, a ideia de que a matemática permite a filosofia operar de maneira mais clara e nítida, uma visão que é negada pela tradição continental. Assim, dado a sua introdução a filosofia por outra área, Lawvere parece não ser afetado pela dicotomia analítica/continental, algo que, ao nosso ver, é um sinal de "maturidade filosófica", ou seja, a compreensão de que, possivelmente, ambos os lados contam versões diferentes (e igualmente importantes) da mesma história.

A distinção de Hegel entre lógica objetiva e subjetiva é exposta na seguinte passagem:

O que deve ser considerado é toda a Noção, primeiramente como a Noção sob a forma de 
sendo, em segundo lugar, como a Noção; no primeiro caso, a Noção é apenas em si mesma, a Noções de realidade ou de ser; no segundo caso, é a Noção como tal, a Noção existente para si mesma...Assim, a lógica deve ser dividido principalmente na lógica da Noção como sendo e da Noção como Noção ou, empregando os termos habituais em lógica "objetivd" e "subjetivd" (HEGEL, 2010, p. 79).

A Noção de Hegel pode ser compreendida como uma categoria que compreende tanto:

(i) a realidade (ser);

(ii) pensar sobre a realidade (ser a pensar).

Esta noção forma o idealismo objetivo de Hegel, dividindo a lógica em (i), a lógica do Ser, que pode ser comparada com uma espécie de ontologia, e (ii) a lógica do pensar, que é o que comumente chamamos de lógica. A lógica objetiva de Hegel, ou ontologia hegeliana, não é apenas limitada a uma noção de metafísica, como a lógica transcendental de Kant, que para Hegel, da pouca importância para a parte objetiva e focasse apenas na porção subjetiva da lógica. Como Hegel coloca:

Recentemente, Kant opôs-se ao que normalmente é chamado de outra lógica, ou seja, uma lógica transcendental. O que aqui tem sido chamado de lógica objetiva corresponderia em parte ao que com ele é a lógica transcendental. Kant a distingue do que ele chama lógica geral desta forma, a que trata das noções que se referem a priori aos objetos, e consequentemente não abstrai de todo o conteúdo da cognição objetiva, ou, em outras palavras, contém as regras do 
pensamento puro de um objeto, e, ao mesmo tempo, trata da origem da nossa cognição [...]. É a este segundo aspecto que o interesse filosófico de Kant é dirigido exclusivamente (HEGEL, 2010, p. 81).

Dado a passagem acima podemos fazer a seguinte comparação: a lógica objetiva de Hegel "corresponde em parte" à lógica transcendental de Kant, ou, a lógica transcendental de Kant é uma "aproximação" da lógica objetiva de Hegel. Para Hegel, Kant diferencia a lógica transcendental da "lógica geral" pelo fato da lógica transcendental não ser totalmente não ambígua ao tópico, o que para Kant resulta na implicação de que a lógica geral é aplicável na matemática e ciências empíricas, mas não ao transcendental. Assim a interpretação do transcendental seria uma "partidd" da lógica para a metafísica.

Uma das ideias centrais da tese hegeliana é continuar fazendo algo como lógica, ao invés de ubstr para uma física especulativa (metafísica), tornemos a objetiva. Hegel compara a sua lógica objetiva com a metafísica tradicional na seguinte ubstra:

A lógica objetiva, então, toma o lugar da antiga metafísica, que se pretendia ser a construção científica do mundo apenas em termos de pensamentos. Se tivermos em conta a forma desta ciência, então ela é a primeira e imediata ontologia cujo lugar é tomado pela lógica objetiva [...] além disso, a lógica objetiva também compreende o resto da metafísica, na medida em que é uma ubstrate de compreender como as formas de pensamentos puros, ubstrates particulares tirados principalmente da concepção de alma [...] porém, considera 
Ágora Filosófica

estes formas livres desses ubstrates, dos sujeitos da concepção, considerando elas, sua natureza e seu valor, em seu próprio caráter.[...]. A lógica objetiva é, portanto, a uma genuína crítica a eles [Kant]- uma crítica aos que os não consideram como contrastados sob as formas abstratas do a priori e do a posteriori...(HEGEL, 2010, p. 85).

Dessa forma, Hegel se livra da distinção a priori versus a posteriori de Kant, o que de certa forma diminui o papel dos dados empíricos nas ciências, e sugere que a construção de teorias sobre o mundo, possam ser feitas só em termos de "pensamentos", numa nova forma dialéctica. O preço de se usar deste método sem comprometer o caráter empírico da ciência, é motivo de crítica a tese hegeliana de lógica objetiva.

A à lógica subjetiva, para Hegel, é a segunda etapa de um único processo de raciocínio dialético, que é constituído por três partes: a lógica do Ser, a lógica da Essência e a lógica da Noção, a última parte sendo chamada de "objetivação da lógicd". Para Hegel a distinção entre lógica objetiva e a subjetiva deve ser pensada como disputável, ou seja, sujeita à polêmica, pois, em sua tese o que realmente é visado é não a demarcação que divide ambas, mas as transformações que interagem entre ambas resultando na lógica da Noção (HEGEL, 2010, p. 86). A forma da filosofia hegeliana é refletida nos objetivos de Lawvere, que busca reformular a lógica dialética de Hegel em termos matemáticos pela lógica categórica, e utilizar a lógica dialética de Hegel como guia pesquisa matemática. O objetivo final de Lawvere é análogo a objetificação da lógica, a lógica da Noção de Hegel (LAWVERE, 1992).

Lawvere (1993) distingue entre a lógica objetiva e a 
subjetiva no contexto da lógica categórica da seguinte forma: lógica objetiva - um guia para as construções complexas, mas não-arbitrárias, dos conceitos e suas interações que crescem, partir das necessidades da geometria, do estudo do espaço e da quantidade. Podemos substituir o conceito acima de "espaço e a quantidade", por "qualquer objeto de estudo sério", o que, como Hegel, define para Lawvere a negação da lógica objetiva na perspectiva de Kant, pois, o conceito de espaço e a quantidade penetram em qualquer tipo de estudo científico, empírico ou não. Já a lógica subjetiva; trata da inferência entre as proposições, sendo de interesse apenas aquelas que concretizam os conceitos, ou seja, os objetificam.

Enquanto Lawvere relaciona sua lógica objetiva com categorias de espaço e quantidade, Hegel desenvolve todas as categorias, após desenvolvimento dialético, a partir das categorias de Ser, Nada e Tornar-se. Lawvere defini, a partir dos conceitos de "espaço" e "quantidade", um "guia teórico de categoria para construções conceituais que crescem de qualquer campo de estudo sério". No sentindo filosófico, os conceitos de espaço e quantidade, como para Hegel, o Ser e o Nada (os opostos), permeiam qualquer campo de estudo "sério", o que podemos interpretar como empírico, de modo que a lógica da Noção não se restringe a especificidades.

Num ponto de vista partilhado por ambos Lawvere e Hegel, o conceito de magnitude kantiana (KANT, 1763[1992]), que permite a nítida distinção entre as construções matemáticas úteis e as matemáticas "metafísicas", ou "especulativas", é uma proposta duvidosa. Wigner (1960) em seu artigo seminal " $A$ eficácia irrazoável da matemática nas ciências naturais" expressa 
essa ponto no sentido de que, dado a magnitude e abrangência da matemática, e sua capacidade de lidar com conceitos totalmente abstratos, é fantástica sua eficiência e funcionalidade em explicar fenômenos naturais. É importante ressaltar que muitas contribuições que a matemática pura teve ha matemática aplicada foram inspiradas por motivos, talvez para Kant, de interesse metafísico (abstrato) total. Assim, considerar que apenas a "matemática aplicadd" é relevante as ciências naturais, é uma contradição, pois, a fonte de toda teoria aplicável é a sua essência pura e abstrata.

Lawvere promove a ideia de que as leis da lógica não são autossustentáveis, mas sim apenas uma forma de metafísica possível. O que o movimento positivista no início do século XX procurou fazer foi reavivar uma metafísica fundamentada apenas na lógica de primeira ordem (proposicional) e segunda ordem (Teoria dos Grupos) (RUSSEL, 1918), e inferindo que está seria a metafísica "real', ou, a não-especulativa. Contudo, o problema de aterramento lógico permanece em aberto. A objetificação da lógica de Hegel, formalizada em parte por Lawvere, trata-se de uma possível estratégia para resolver o problema de aterramento lógico em metafísica.

Para Lawvere (2005), “a lógica é um caso especial de geometrid', este pensamento vai em total oposto ao pano de fundo do Método Axiomático de Hilbert, onde a lógica fornece uma base às teorias matemáticas. A dialética axiomática de Lawvere nos sugere que a geometria, e não a lógica, é o aspecto principal, e que a lógica é um tipo especial de geometria. No sentido hegeliano, a geometrização da lógica de Lawvere pode ser filosoficamente comparada com uma forma de objetivação da lógica, resultando na lógica da Noção de 
Hegel. Contudo, apenas como uma aproximação, ou, uma resultado corolário da tese hegeliana. Para Hegel (2010) a lógica subjetiva emerge do objetivo, na interpretação de Lawvere a lógica objetiva é primariamente construída por categorias objetivas empiricamente significativas, ou seja, que lidem com noções de espaço e quantidade.

Contudo, ainda podemos nos indagar sobre qual modelo lógico deveria ser o "mais verdadeiro", ou qual metafísica melhor representa a física real? Possivelmente, tais questões sejam mais direcionadas a filosofia e a metamatemática, e não com a matemática em si. O que a matemática pode fazer é escolher seu ponto de vista, seu sistema lógico, e pragmaticamente realizar seu trabalho com base nele, mas o que Lawvere nos sugere e que estás questões são, na verdade, totalmente matemáticas! Pelo uso da lógica categórica, Lawvere objetifica a lógica como Hegel, e, de certa forma, a aterra e a torna manipulável, podendo ser analisada intrinsecamente, dentro de um determinado topos (universo matemático), ou extrinsecamente como a conexão entre diferentes topoi (universos matemáticos).

A contribuição de Lawvere para a Axiomatização da lógica e matemática categórica inspirada pela filosofia hegeliana, dentro do contexto axiomático formal proposto por Hilbert seria impossível. A ideia de Lawvere de internalização e objetificação da lógica categórica reaparece numa interpretação geométrica em áreas da matemática que tiveram sua origem, de certa forma, na matemática categórica, especificamente na topologia, na Teoria dos Tipos, e finalmente na Teoria dos Tipos Homotópica, que tem sido proposta como a nova linguagem para os fundamentos da matemática. 


\section{Teoria de tipos homotópica}

A Teoria de Tipos Homotópica pode ser utilizada como uma linguagem fundamental para a matemática, ou seja, uma alternativa à Teoria dos Conjuntos ZermeloFraenkel, ambas possuem várias distinções importantes. A teoria dos conjuntos possui duas "camadas" distintas:

- o sistema dedutivo, que envolve lógica de primeira (proposicional);

- a segunda camada são as formulações criadas dentro deste sistema, os axiomas de ZermeloFraenkelsendo, a linguagem formal, padrão, da matemática.

Dessa forma a Teoria dos Conjuntos ZermeloFraenkel trata-se sobre a interação entre os objetos da segunda camada (conjuntos) e os objetos da primeira camada (proposições). Enquanto isso, a Teoria de Tipos Homotópica é o seu próprio sistema dedutivo, não precisando ser formulada dentro de qualquer subestrutura como lógica de primeira ou segunda ordem, e, diferente da distinção básica de proposições e conjuntos da teoria dos conjuntos, a teoria de tipos homotópica possui apenas uma noção básica: tipos (ACZEL et al. 2013, p. 17).

Todas estas abordagens podem ser vistas como diferentes desenvolvimentos das propostas axiomáticas de Lawvere em 1970 sobre a internalização da lógica categórica e a sua natureza geométrica. Em 2006 essas propostas culminaram com Voevodsky (2011), ao publicar uma nota inédita onde segeriu um grande programa de investigação de sistemas de tipos por métodos homotópicos. A ambição do programa de pesquisa proposto por Voevodsky seria construir novos 
fundamentos para a matemática, que ele chama Fundações Univalentes. Nas palavras de Voevodsky:

A ampla motivação por trás das fundações univalentes é o desejo de ter um sistema em que a matemática possa ser formalizada de uma forma tão natural quanto possível. Embora seja possível codificar toda a matemática na teoria de Zermelo-Fraenkel, a forma como isso é feito é frequentemente feia; pior ainda, quando se faz isso, continuam a existir muitas afirmações em Zermelo-Fraenkel que são matematicamente sem sentido. Este problema torna-se particularmente presente na tentativa de formalizar a matemática por computadores; nos fundamentos padrões, escrever por completo até as deduções mais básicas, como isomorfismo entre conjuntos, ou à estrutura de grupo sobre um conjunto, requer muitas páginas de símbolos. Fundamentos univalentes procuram melhorar esta situação através de um sistema, baseado na teoria de tipo homotópica[...] (VOEVODSKY, 2011, p. 7).

Além do seu aspecto teórico, esta iniciativa tem um objetivo extremamente prático: permitir uma utilização sistemática de assistentes informáticos como o Coq, na prática matemática cotidiana, Coq é um programa de computador interativo que permite a expressão de afirmações matemáticas, como também na verificação e produção de provas e teoremas matemáticos (GONTHIER, 2008). As fundações univalentes pretendem proporcionar a matemática contemporânea uma linguagem universal que tenha um aspecto formal, intuitivo, e programável.

Quando comparamos a formalização proposta por Voevodsky com o método axiomático formal de Hilbert, 
vemos além de algumas diferenças chaves, uma aproximação ao que Lawvere citou como "uma fundamentação da matemática ligada com a sua prática".

Em ambos os casos temos uma sintaxe simbólica e uma interpretação desta sintaxe, mas as relações entre a sintaxe e a sua interpretação não são as mesmas. No caso da interpretação de Hilbert, o modelo dualista funciona separando os conceitos lógicos (subjetivos) e os grupos (objetivos), enquanto isso, a abordagem de Voevodsky não envolve a mesma diferença entre categorias lógicas e não lógicas. O intuito de Voevodsky é de fundir às duas categorias, e lógica objetiva e a lógica subjetiva em termos hegelianos, o mais próximo possível por sua vez, onde tanto os símbolos lógicos e as mesmas construções são interpretadas tanto em termos lógicos como geométricos, a dialética é estabelecida de uma forma totalmente internalizada, cujo, resultado final poder ser análogo a objetificação, aqui usado o termo internalização, da lógica.

Estas características tornam o Método Axiomático de Voevodsky significativamente diferente do Método Axiomático de Hilbert, e promovem uma nova maneira de interpretar a matemática. O projeto das fundações univalentes continua em aberto, sendo uma área de intensa pesquisa e desenvolvimento na matemática, com a promessa de fazer as fundações dessa disciplina mais simples, acessíveis, dialéticas e intuitivas, algo que com o auxílio de ferramentas de augmentação matemática como Coq, entre outros programas similares, promete impulsionar a matemática para uma nova era.

\section{Conclusão}

A lógica categórica sugere uma estratégia de unificação muito diferente da axiomatização proposta por 
Hilbert. Enquanto o método axiomático formal (Teoria dos Conjuntos Zermelo-Fraenkel) utiliza uma estrutura invariante central, a lógica de primeira ordem, supostamente partilhada por todos os espaços geométricos, ela organiza o seu universo em termos de mapas/objetos, lógica e conjuntos. Já na proposta das Fundações Univalentes, está regra deontológica universal, de ter a lógica de primeira ordem como estrutura invariante, é abandonada e convertida em uma forma de pluralismo lógico, onde o Método Formal Axiomático é apenas um caso especial de sua generalidade.

As abordagens axiomáticas de Lawvere, cuja influência pode ser rastreadas desde a matemática e lógica categórica, na metade do século XX, até o século XXI, com Voevodsky e as Fundações Univalentes da Matemática partilham de uma similaridade, se não a mesma fonte inspiradora. Está fonte diz respeito à relação entre a geometria e a lógica nas suas teorias, Lawvere em seus termos, descreve estas relações em termos hegelianos como uma contradição dialéctica. Ironicamente, o idealismo lógico de Hegel, criticado pelo pensamento positivista liderado por Russel, Hilbert e seus contemporâneos, foi algo extremamente importante para rejuvenescer e aprimorar as fundações da própria matemática.

A abordagem de Hilbert, e o modelo axiomático formal em si, assume uma relação assimétrica entre geometria e lógica, entre a maneira de pensar e o objeto de interesse, a geometria sendo supostamente lógica enquanto a lógica não é geométrica. As abordagens de Lawvere, inspiradas pelo conceito de equivalência como uma forma de transformação, a unidade dos opostos de Hegel, tornam esta relação simétrica: as suas teorias 
geométricas são lógicas, e a sua lógica é geométrica.

\section{Referências}

ACZEL, P. AHRENS, B. ALTENKIRCH, T. AWODEY, S. BARRAS, B. BAUER, A. BERTOT, Y. BEZEM, M. COQUAND, T. FINSTER, E. GRAYSON, D. HERBELIN, H. ANDRE, J. LICATA, D. LUMSDAINE, P. MAHBOUBI, A. MARTIN-LÖF, P. MELIKHOV, S. PELAYO, A. SOLOVIEV, S. Homotopy Type Theory: Univalent Foundations of Mathematics. The Univalent Foundations Program. Institute for Advanced Study, Princeton. 2013.2 Disponível em:

https://arxiv.org/pdf/1308.0729.pdf

ALLEN, H. Algebraic topology. Cambridge: Cambridge University Press. p. 185, 2002. ISBN 9780521795401.

BOURBAKI, N. Architecture of mathematics. The American Mathematical Monthly, 57(4), p. 221-232, 1950.

CANTOR, G. Ueber eine elementare Frage der Mannigfaltigkeitslehre. Jahresbericht der Deutschen Mathematiker-Vereinigung, 1, p. 75-78, 1892.

CARNAP, R. The Elimination of Metaphysics Through Logical Analysis of Language. Erkenntnis Vol II, 1932.

COHEN, P. J. Set theory and the continuum hypothesis. Mineola, New York: Dover Publications, 2008 [1966]. ISBN 978-0-486-46921-8.

COHEN, P. J. The independence of the continuum hypothesis. Proceedings of the National Academy of Sciences U.S.A., 50, p. 1143-1148, 1963.

EILENBERG, S. SAUNDERS, M. General theory of natural equivalences. Trans. Amer. Math. Soc. 58, p. 231-294, 1945. DOI: https://doi.org/10.1090/S0002-9947-1945$\underline{0013131-6}$

GARSON, J. Modal Logic. Stanford Encyclopedia of 
Philosophy. First published Tue Feb 29, 2000; substantive revision Sat Sep 8, 2018. Disponível em: https://plato.stanford.edu/entries/logicmodal/\#PosWorSem

GÖDEL, K. The consistency of the axiom of choice and of the generalized continuum hypothesis. Proceedings of the National Academy of Sciences U.S.A., 24, p. 556-557, 1938. GONTHIER, G. Formal Proof-The Four-Color Theorem. Notices of the American Mathematical Society, 55 (11), pp. 1382-1393, 2008. MR 2463991

HEGEL, G.W.F. Science Of Logic. Translated by MILLER, A.

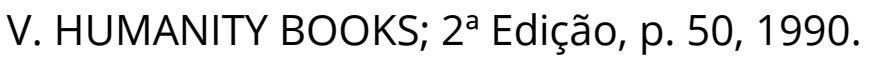

HEGEL, G.W.F. The Science of Logic [Wissenschaft der LogikJ, translated by DI GIOVANNI, G. New York: Cambridge University Press, 2010.

HEGEL, G.W.F. The Science of Logic. Translated by George di Giovanni. Cambridge University Press, 2010.

HILBERT, D. Grundlagen der Geometrie. Leipzig, 1899.

HILBERT, D. The new grounding of mathematics. W. Ewald (ed.), From Kant to Hilbert: A Source Book in the Foundations of Mathematics, 2, p. 1115-1134, 1996.

HILBERT, D. Axiomatic thought. W. Ewald (ed.), From Kant to Hilbert: A Source Book in the Foundations of Mathematics, 2, p. 1105-1115, 1996.

HILBERT, D. Mathematical problems. Bulletin of the American Mathematical Society, 8(10), p. 437-479, 1902.

KAN, D. M. Adjoint Functors. Transactions of the American Mathematical Society. 87 (2), p. 294-329, 1958. doi:10.2307/1993102.

KANT, I. MEERBOTE, R. Attempt to introduce the concept of negative magnitudes into philosophy (1763). In D. Walford (Ed.), Theoretical Philosophy, p. 1755-1770 (The Cambridge Edition of the Works of Immanuel Kant, pp. 
203-206). Cambridge: Cambridge University Press, 1992. KUNEN, K. Set Theory: An Introduction to Independence Proofs. Elsevier, 1980.

LAWVERE, F.W. Categories of space and quantity. J. Echeverria et al (eds.), The Space of mathematics, de Gruyter, Berlin, New York, p. 14 - 30, 1992.

LAWVERE, F.W. Cohesive Toposes and Cantor's "lauter Einsen". Philosophia Mathematica, The Canadian Society for History and Philosophy of Mathematics, Series III, Vol. 2, p. 5-15, 1994.

LAWVERE, F.W. Comments on the Development of Topos Theory. Development of Mathematics 1950-2000, J.-P. Pier (Ed) Birkhäuser Verlag, Basel, p. 715-734, 2000.

LAWVERE, F.W. ROSEBRUGH, R. Sets for Mathematics. Cambridge University Press, 2003.

LAWVERE, F.W. Some thoughts on the future of category theory. In: Carboni A., Pedicchio M.C., Rosolini G. (eds) Category Theory. Lecture Notes in Mathematics, vol 1488. Springer, Berlin, Heidelberg, 1991.

LAWVERE, F.W. Taking categories seriously. Reprints in Theory and Applications of Categories, 2, p. 1 - 24, 2005.

LAWVERE, F.W. Tools for the advancement of objective logic: closed categories and toposes. J. Macnamara and G.E. Reyes (Eds.), The Logical Foundations of Cognition, Oxford University Press 1993 (Proceedings of the Febr. 1991 Vancouver Conference "Logic and Cognition"), p. 4356, 1994.

LEWIS, D. Counterfactuals. Blackwell Publishers, Massachusetts, USA, 1973.

PEIRCE, C. S. On the Logic of Number». American Journal of Mathematics. 4, p. 85-95, 1881. doi:10.2307/2369151 REICHENBACH, $\mathrm{H}$. The Rise of Scientific Philosophy. Berkeley \& Los Angeles: University of California Press, p. 
$72,1951$.

RODIN, A. Axiomatic Method and Category Theory. 2012. DOI 10.1007/978-3-319-00404-4.

https://arxiv.org/pdf/1210.1478.pdf

RUSSELL, B. The philosophy of logical atomism. The Monist, 28, p. 495-527, 1918.

SULLIVAN, A. Logicism and the Philosophy of Language: Selections from Frege and Russell. Broadview Press, Logic As The Essence Of Philosophy, p. 277, 2003. ISBN-13: 9781551114712

VOEVODSKY, V. A. Univalent foundations. Mathematisches Forschungsinstitut Oberwolfach, Mini-Workshop: The Homotopy Interpretation of Constructive Type Theory, Report N 11/2011, p. 7-10, 2011.

WIGNER, E. P. (1960). The unreasonable effectiveness of mathematics in the natural sciences. Richard Courant lecture in mathematical sciences delivered at New York University, Communications on Pure and Applied Mathematics, 13, p. 1-14, 1959. DOI:10.1002/cpa.3160130102.

ZERMELO, E. Untersuchungen über die Grundlagen der Mengenlehre /. Mathematische Annalen. 65, pp. 261-281, 1908. doi:10.1007/BF01449999.

Nicholas Kluge Corrêa

Mestre em Engenharia Elétrica (Escola Politécnica, PUCRS), Doutorando em Filosofia (PUCRS). Bolsista CAPES/PROEX.

E-mail: nicholas.correa@acad.pucrs.br

Submetido: $17 / 07 / 2020$

Aprovado: 28/09/2020 